\title{
Structure and Mechanical Properties of Sequential Interpenetrating Polymer Networks III. Poly(acrylonitrile-co-butadiene)/ Poly(methyl methacrylate) System
}

\author{
Hiroshi ADACHI and Tadao KoTAKA* \\ Manufacturing Development Laboratory, Mitsubishi, Electric Corporation, \\ Amagasaki, Hyogo 661, Japan \\ * Department of Macromolecular Science, Faculty of Science, \\ Osaka University, Toyonaka, Osaka 560, Japan
}

(Received September 28, 1982)

\begin{abstract}
Two types of interpenetrating polymer networks (IPNs) consisting of incompatible poly(acrylonitrile-co-butadiene) (NBR) and poly(methyl methacrylate) (PMMA) as the first and second components, respectively, were prepared: One was semi-IPNs in which only NBR was crosslinked, and the other was full-IPNs in which both NBR and PMMA were crosslinked. Electron micrographs showed heterogeneous and fairly homogeneous structures containing a continuous NBR phase for semi- and full-IPNs, respectively. These structures were compatible with the mechanical properties (viscoelastic properties and stress-strain behavior). The elastic moduli of these IPNs may be predicted by the Davies model which takes particle-particle interactions into account. The difference in the extent of molecular mixing of the two components of the semi- and full-IPNs may be explained by the formation of the microgels of the second component and the crosslinks in each component.
\end{abstract}

KEY WORDS Poly(acrylonitrile-co-butadiene)/ Poly(methyl methacrylate)

/ Sequential Interpenetrating Polymer Networks / Microgel / Crosslinks /

Electron Micrograph / Viscoelastic Properties / Stress-Strain Behavior /

Interpenetrating polymer networks (IPNs) are a special type of polymer blends designed to obtain compatible materials from polymer components either mutually miscible or immiscible. ${ }^{1-4}$ One of the most common IPNs is the so-called sequential IPNs (SIPNs), and can be synthesized by swelling a preformed network of polymer (I) with a second monomer (II) containing a crosslinker and an initiator and then allowing the monomer (II) to polymerize in situ. If either polymer (I) or polymer (II) is not crosslinked, we call the IPN a semi-IPN. On the other hand, if both components are crosslinked, we call the IPN a full-IPN.

We carried out a study to elucidate the synthesismorphology-property relationship of SIPNs in greater detail, and reported experimental data on two SIPNs, one consisting of a semicompatible polymer pair: poly(ethyl acrylate) (PEA) and poly(methyl methacrylate) (PMMA), ${ }^{5}$ and the other consisting of complex forming polymer pair: polyoxyethylene (POE) and poly(acrylic acid) (PAA). ${ }^{6}$ The results obtained for the semicompatible pair are as follows: Microgels first appeared in the preformed PEA network from the MMA mixture and were eventually interconnected with one another to form a SIPN which had a mutually interpenetrating continuous phase. We compared the properties of semi- and full-IPNs of PEAy PMMA, and found that dynamic behavior under a small amplitude oscillation and stress relaxation at a fixed small strain was linear viscoelastic for both semi- and full-IPNs. However, for large and high speed deformation, the two IPNs showed significantly different behavior owing to the difference in effective network chain density.

For the complex forming pair, it was found that SIPNs with the PAA content between 30 and 60 $\mathrm{mol} \%$ were single-phase systems. In those having 
either lower or higher PAA content, semicrystalline POE or amorphous PAA microdomains were phase-separated from the complex phase. The extent of microphase separation was less in full-IPNs than in semi-IPNs.

A large number of studies on SIPNs of incompatible polymer pair ${ }^{3,4,7}$ showed that the molecular mixing in full-IPNs was more extensive than that in semi-IPNs. ${ }^{7}$ Thus, we prepared semi- and full-IPNs consisting of incompatible poly(acrylonitrile-cobutadiene) (NBR) and PMMA as the (I) and (II) components, respectively, and examined the effect of crosslinking of the second component on the morphology of SIPN by electron microscopy and mechanical tests (viscoelastic behavior and tensile behavior).

\section{EXPERIMENTAL}

Materials. An emulsion polymerized NBR sample with an acrylonitrile content of $29 \%$ by weight was kindly supplied by Japan Synthetic Rubber Co. The sample was crosslinked with $2 \mathrm{wt} \%$ dicumyl peroxide at $150^{\circ} \mathrm{C}$ under $3.5 \mathrm{MPa}$ for $5 \mathrm{~min}$. The crosslinked NBR films had an effective network chain density $v$ of $1.4 \times 10^{2} \mathrm{~mol} \mathrm{~m}^{-3}$ (details reported previously ${ }^{8}$ ). These films were used as the (I) network for the subsequent IPN preparation.

In preparing a full-IPN, the NBR film was allowed to swell in an MMA monomer solution containing $0.1 \mathrm{~mol} \%$ ethyleneglycol dimethacrylate (EGDMA) and $1 \mathrm{wt} \%$ 2,2'-azobisisobutyronitrile
(AIBN) as a crosslinking agent and an initiator, respectively, for a prescribed period of time. The swollen film was placed in an air-tight polymerization cell, and allowed to stand at room temperature for $30 \mathrm{~min}$, and then heated at $70^{\circ} \mathrm{C}$ for $180 \mathrm{~min}$ to complete the PMMA network formation. The full-IPN specimen was then dried in vacuo at $60^{\circ} \mathrm{C}$ for 4 days. The weight loss was $2-3 \%$. The specimen was subjected to extraction by refluxing benzene for $6 \mathrm{~h}$. The extractable fraction was less than $5 \%$ for all samples.

For the semi-IPN preparation, the same procedure as used in the full-IPN preparation was employed, except that the MMA monomer mixture contained only $1 \mathrm{wt} \%$ AIBN and no EGDMA. Since hydrocarbon radicals produced from the decomposition of AIBN do not very actively attack the NBR chain, ${ }^{9}$ there was little concern about the grafting of PMMA (II) component onto the NBR (I) component. Table I shows the sample characteristics of the semi- and full-IPNs. All specimens, except the semi-IPN S-74 with the highest PMMA content, were transparent.

Methods. Morphology was observed by a transmission electron microscope (Japan Electron Optics Laboratory Co. Ltd.), with the sample treated by the osmium tetroxide $\left(\mathrm{OsO}_{4}\right)$ hardening and staining technique developed by Kato. ${ }^{10}$ Since an excessively long time was required for $\mathrm{OsO}_{4}$ staining of the NBR phase with an aqueous $\mathrm{OsO}_{4}$ solution because of the slow diffusion of $\mathrm{OsO}_{4}$ into the NBR

Table I. Characteristics of NBR/PMMA IPN specimens ${ }^{a, b}$

\begin{tabular}{|c|c|c|c|c|c|}
\hline \multicolumn{3}{|c|}{ Semi-IPN } & \multicolumn{3}{|c|}{ Full-IPN } \\
\hline \multirow[t]{2}{*}{ Code } & $\begin{array}{l}\text { PMMA } \\
\text { content }\end{array}$ & \multirow{2}{*}{$\begin{array}{l}\text { Optical } \\
\text { clarity }\end{array}$} & \multirow{2}{*}{ Code } & $\begin{array}{l}\text { PMMA } \\
\text { content }\end{array}$ & \multirow{2}{*}{$\begin{array}{l}\text { Optical } \\
\text { clarity }\end{array}$} \\
\hline & $w t \%$ & & & $\mathrm{wt} \%$ & \\
\hline S-22 & 22.4 & Clear & F-28 & 27.7 & Clear \\
\hline S-30 & 30.0 & Clear & F-32 & 32.3 & Clear \\
\hline S-52 & 52.1 & Clear & F-52 & 52.3 & Clear \\
\hline S-61 & 60.8 & Clear & F-63 & 62.8 & Clear \\
\hline S-74 & 73.9 & Opaque & F-72 & 72.4 & Clear \\
\hline
\end{tabular}

a The effective network chain density $v$ of the NBR (I) network was $1.4 \times 10^{2} \mathrm{~mol} \mathrm{~m}^{-3}$; the MMA mixtures contained $1 \mathrm{wt} \%$ AIBN and $0.1 \mathrm{~mol} \%$ EGDMA for full-IPN, and $1 \mathrm{wt} \%$ AIBN and without EGDMA for semi-IPN, and polymerized at $90^{\circ} \mathrm{C}$ for $180 \mathrm{~min}$.

b The number in the sample code represents the approximate PMMA content (wt $\%$ ). 
phase, the staining was carried out with an $1 \%$ $(\mathrm{w} / \mathrm{v})$ aqueous solution containing $15 \mathrm{vol} \%$ tetrahydrofuran. ${ }^{11}$

Dynamic mechanical measurements were made by a Rheovibron DDV-II (Toyo Baldwin Co., Tokyo) at a frequency of $11 \mathrm{~Hz}$ and at a heating rate of $1 \mathrm{~K} \mathrm{~min}^{-1}$ in a temperature range from 150 to $450 \mathrm{~K}$. Static mechanical properties (tensile stressstrain behavior and tensile stress-relaxation) were measured with a tensile tester (Iwamoto Seisakusho, Kyoto) equipped with a cryostatic sample chamber which allowed the temperature regulation within $\pm 0.3 \mathrm{~K}$. Stress-strain tests were made at strain rates of from $10^{-1}$ to $100 \% \mathrm{~s}^{-1}$. Tensile stress-relaxation tests were performed in a temperature range from 333 to $413 \mathrm{~K}$. The strain was 0.02 at low temperature and 0.04 in the rubbery region. The initial strain rate was $390 \% \mathrm{~s}^{-1}$. The stress was monitored over a period of $10^{4}$ seconds.

\section{RESULTS}

Morphology. Figure 1 shows the electron micrographs of S-74 and F-72 specimens. As clearly shown by Figure 1a, the S-74 specimen has a heterogeneous structure with the NBR (I) matrix phase. The PMMA component exists in large spherical domains of $5000 \mathrm{~A}$ in diameter and also in smaller domains of a few hundred $\AA$ in diameter. The rest of PMMA component is finely dispersed in the NBR phase. At the boundary of the PMMA domains of $5000 \AA$ in diameter, two components are fairly well mixed, and these domains include a finely dispersed NBR component. On the other hand, as shown in Figure 1b, the PMMA component in the F-72 specimen is finely dispersed in the NBR phase, although this is the major component. No distinct phase boundary can be seen. This difference in morphology between semiand full-IPNs is in agreement with the results reported already on other SIPNs consisting of an incompatible polymer pair. ${ }^{7}$

Mechanical Properties. Figure 2 shows the temperature dependence of tensile storage moduli $E^{\prime}$, loss moduli $E^{\prime \prime}$ and loss tangents $\tan \delta$ for fullIPNs. The $E^{\prime \prime}$ curve for any sample exhibits a peak at a temperature near $250 \mathrm{~K}$, which is higher by $10 \mathrm{~K}$ than the peak temperature of NBR, and a shoulder at a temperature above $250 \mathrm{~K}$. As the
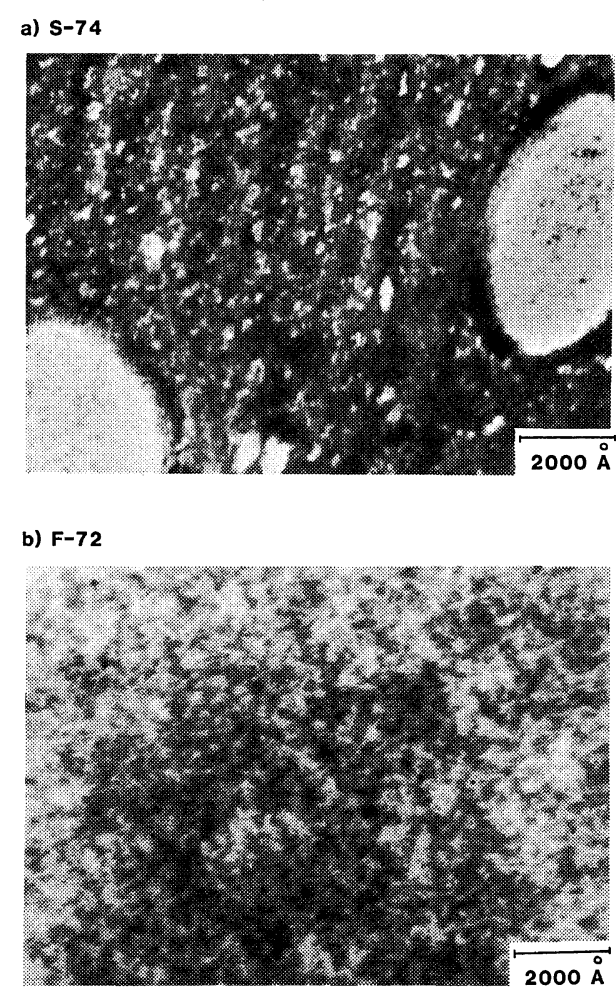

Figure 1. Electron micrographs of (a) semi- and (b) full-IPNs, S-74 and F-72, respectively.

PMMA content increases beyond $50 \mathrm{wt} \%$, the loss peak at $250 \mathrm{~K}$, which may be identified as $T_{\mathrm{g}}$ of the NBR component, diminishes with no accompanying change in loss maximum temperature $T_{\max }$. Even in the IPN containing $72 \mathrm{wt} \%$ PMMA, the loss peak at $250 \mathrm{~K}$ is still apparent. This indicates the existence of a single NBR phase. No loss peak corresponding to $T_{\mathrm{g}}$ of PMMA component was observed. However, a shoulder appeared in the temperature range from $300-450 \mathrm{~K}$ and became larger as the PMMA content increased. This behavior implies that the microdomains of the PMMA component do not grow larger than a few hundred $\AA,{ }^{12}$ and is in agreement with the electron micrograph of Figure 1b. As the PMMA content increases, the $E^{\prime} v s$. temperature curve changes from concave to convex with a sharp decrease at $250 \mathrm{~K}$ and a gradual decrease over the temperature range from 250 to $450 \mathrm{~K}$. The $\tan \delta$ peak of the NBR component shrinks but it sposition does not change, while another peak around $400 \mathrm{~K}$ gradually be- 


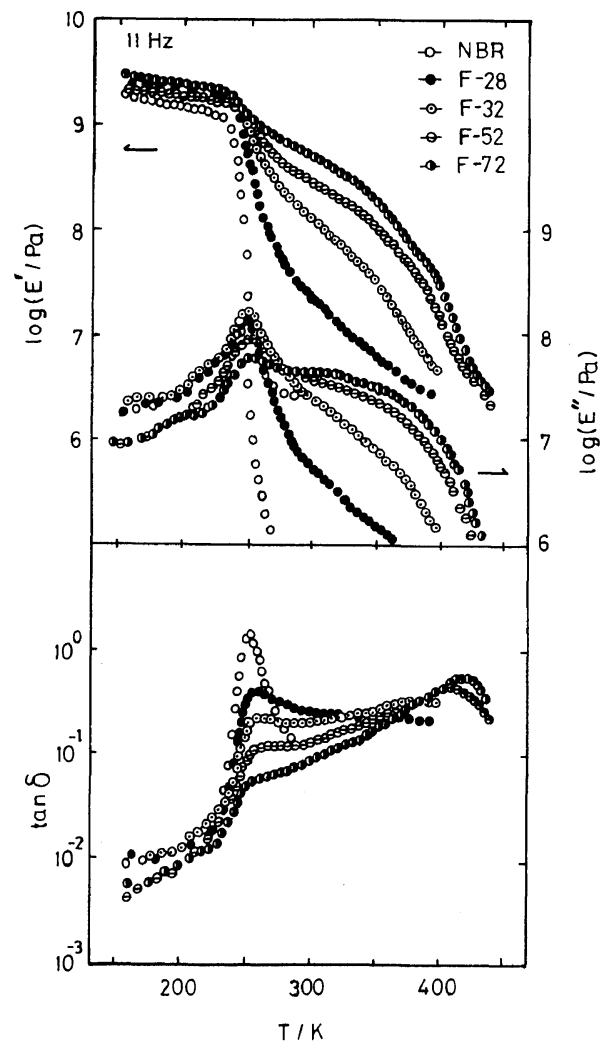

Figure 2. Storage $\left(E^{\prime}\right)$, loss $\left(E^{\prime \prime}\right)$ moduli and loss tangents $(\tan \delta)$ at $11 \mathrm{~Hz}$ plotted against temperature for full-IPNs with various composition, determined at a heating rate of $1 \mathrm{~K} \mathrm{~min}^{-1}$.

comes larger and shifts to higher temperature, as the PMMA content increases. For F-52, the value of $\tan \delta$ is almost constant over the temperature range from 250 to $400 \mathrm{~K}$, which are the $T_{\mathrm{g}}$ of NBR (I) and PMMA (II) components, respectively. The $E^{\prime}, E^{\prime \prime}$, and $\tan \delta$ curves for semi-IPNs, except those for S74, also tend to be similar to those for full-IPNs.

In Figure 3, the $E^{\prime}$ values for semi- and full-IPNs at $300 \mathrm{~K}$ are compared with three mixture rules: the three dimensional Takayanagi model by Kraus and Rollman, ${ }^{13}$ the $1 / 5$ power law model by Davies, ${ }^{14}$ and the logarithmic rule model. These three models can be used to predict Young's modulus of a composite consisting of two continuous phases. ${ }^{15,16}$

In Figure 3, the shaded zone represents the Kraus-Rollman model with the lower and upper bounds. The broken curve is the Davies' $1 / 5$ th power law. The solid straight line shows the loga-

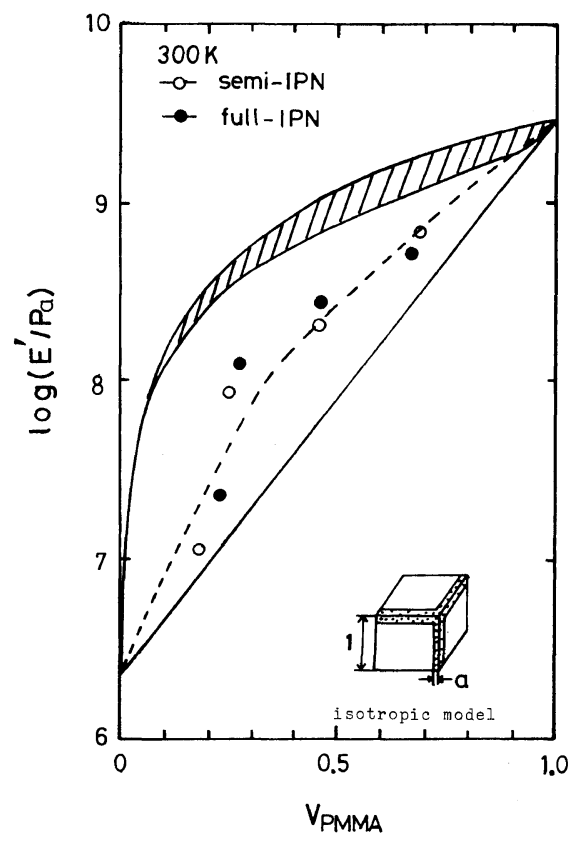

Figure 3. Young's moduli $\left(E^{\prime}\right)$ at $300 \mathrm{~K}$ versus composition in volume fraction of PMMA $\left(V_{\mathrm{PMMA}}\right)$ for semiand full-IPNs. Shaded area indicates the values calculated from the isotropic model, eq 1 and 2, broken curve Davies' $1 / 5$ power model, eq 3 , and solid curve logarithmic mixture rule, eq 4 .

rithmic rule model.

The Kraus-Rollman mode ${ }^{13}$ is an extension of the Takayanagi model ${ }^{17}$ to the three-dimensional case. In the Takayanagi model, the phase-phase interaction is not specifically considered. ${ }^{18}$ The Davies model has been found to hold for IPNs consisting of an incompatible pair of styrenebutadiene copolymer and polystyrene, ${ }^{7}$ and interstitial polymers such as PMMA-modified polyurethane elastomers (a kind of semi-IPN). ${ }^{18}$ Davies et al..$^{18}$ has pointed out that his model gives the best fit to the experimental data, because it takes particle-particle interactions into account. The logarithmic rule may be applied to isotropic binary composites with two continuous phases, such as IPN. ${ }^{19}$

The elastic moduli of the present IPNs fall on the curve predicted by the Davies model. By considering the structure containing the matrix NBR phase, this finding may be regarded as evidence for molecular interactions of the two components in the 
a) semi-IPN

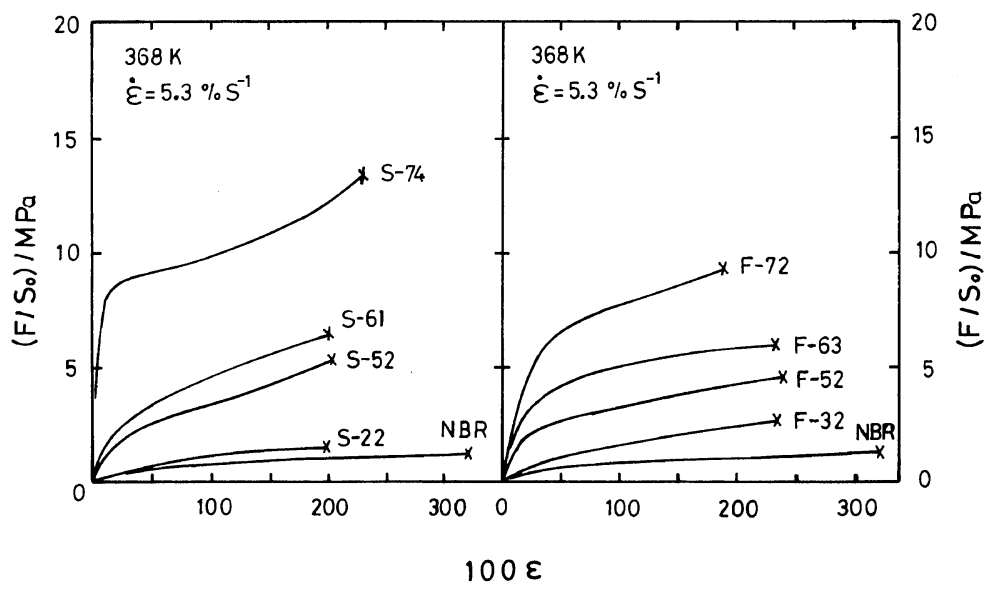

Figure 4. Stress-strain curves at $368 \mathrm{~K}$ for (a) semi- and (b) full-IPNs determined at a strain rate of $5.3 \% \mathrm{~s}^{-1}$. Marks $(x)$ denote break points.

interfacial layer.

Figure 4 shows the composition dependence of stress-strain curves for semi- and full-IPNs at a strain rate of $5.3 \% \mathrm{~s}^{-1}$ at $368 \mathrm{~K}$ just below the $T_{\mathrm{g}}$ of the PMMA (II) component. In the measurements of these curves, the specimens showed no necking but could be extended uniformly without stress whitening. All the specimens became tough and stronger with an increase in PMMA content. However, even the IPN with $c a$. $75 \mathrm{wt} \%$ PMMA content exhibited no yielding phenomenon. The matrix NBR phase dominated the stress-strain behavior.

The mechanical properties described above imply the following. The NBR and PMMA networks are mixed fairly well but not as fully as in the IPNs consisting of a semicompatible pair. ${ }^{5}$ The continuous NBR domains may exist in the specimen of any composition, while the distinct PMMA domains with diameters larger than a few hundred $\AA$ may not exist even in specimens with high PMMA content except for S-74. The PMMA domains are forced to disperse through the specimen and interpenetrate with the NBR network. Their size becomes somewhat larger with increasing PMMA content, but cannot become large enough to make the specimen film turbid, except for S-74.

Comparison of Semi- and Full-IPNs. We now direct our attention to the difference between samples S-74 and F-72, which are semi- and full-IPNs

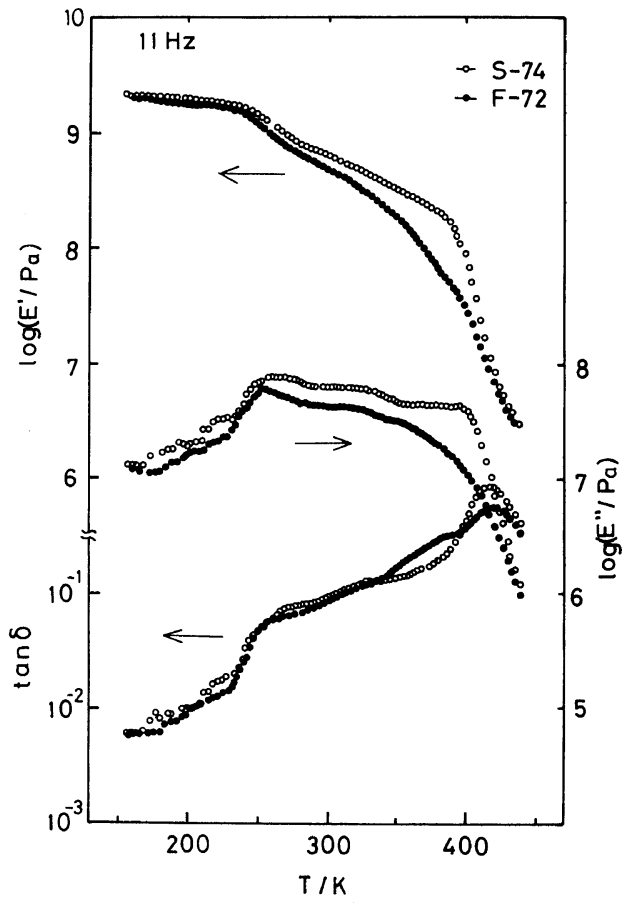

Figure 5. Storage $\left(E^{\prime}\right)$, loss $\left(E^{\prime \prime}\right)$ moduli, and loss $\operatorname{tangents}(\tan \delta)$ at $11 \mathrm{~Hz}$ versus temperature for semiand full-IPNs, S-74 and F-72, respectively, determined at a heating rate of $1 \mathrm{~K} \mathrm{~min}^{-1}$.

having nearly the same PMMA content. Figure 5 shows the temperature dependence of $E^{\prime}, E^{\prime \prime}$, and $\tan \delta$ for these samples. The $E^{\prime \prime}$ curve for S-74 


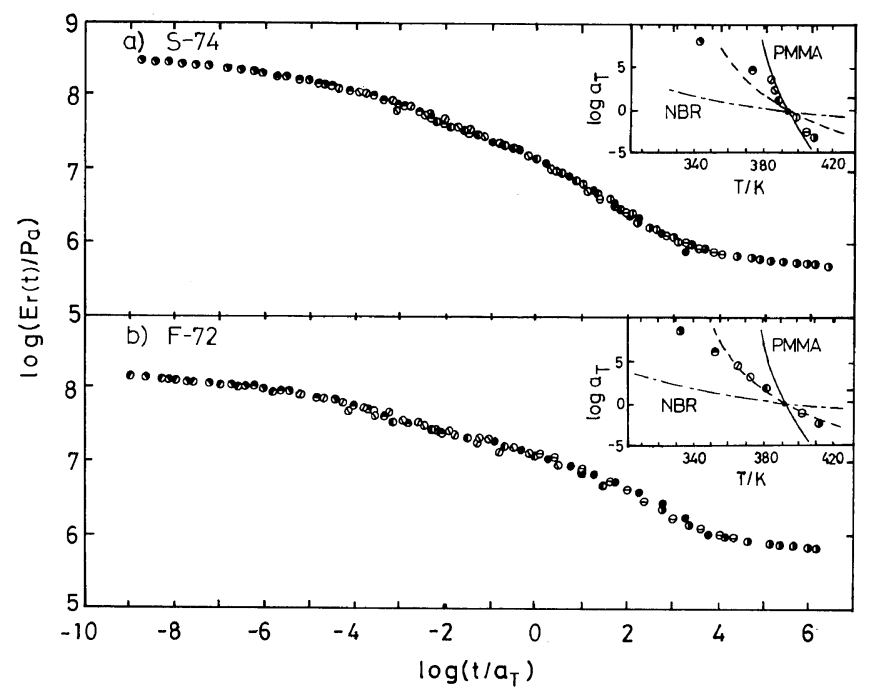

Figure 6. Tensile stress-relaxation master curves for (a) semi-IPN (S-74) and (b) full-IPN (F-72) reduced to $T_{\mathrm{r}}=393 \mathrm{~K}$. Data points include those obtained in the range of strain $0.02-0.04$ over a temperature range from 313 to $413 \mathrm{~K}$. the initial strain rate was $390 \% \mathrm{~s}^{-1}$. Curves in the inserts represent the WLF equations for NBR, PMMA, and IPNs.

shows a distinct shoulder at $400 \mathrm{~K}$, but not for F-72. The $E^{\prime}$ curve for S-74 exhibits a distinct two-step decrease in the range from 250 to $400 \mathrm{~K}$. This difference may be interpreted in terms of the structures of the samples observed electron microscopically. No such difference in dynamic mechanical behavior between semi- and full-IPNs was observed for the semi-compatible system consisting of poly(ethyl acrylate) and poly(methyl methacrylate). ${ }^{5}$

Figures $6 \mathrm{a}$ and $6 \mathrm{~b}$ show the tensile stressrelaxation master curves for S-74 and F-72, respectively, with the reference temperature $T_{\mathrm{r}}$ taken to be $393 \mathrm{~K}$ for both. Both curves show broad transitions, the latter being even broader than the former. The inserts in these figures show log (shift factor $a_{\mathrm{T}}$ ) versus temperature plots, where the broken curves represent the WLF equation ${ }^{20}$ with $T_{\mathrm{r}}=393 \mathrm{~K}$. The $\log a_{\mathrm{T}}$ data for F-72 fall on the WLF curve, except at lower temperatures, while the $\log a_{\mathrm{T}}$ data for S-74 follow the WLF curve not for the IPN but for PMMA except at lower temperatures. In sample S-74 having a microheterogeneous structure, the relaxation of the hard PMMA component surpasses that of the soft NBR component in the temperature region studied. A similar tendency was observed in the dynamic mechanical property described above.

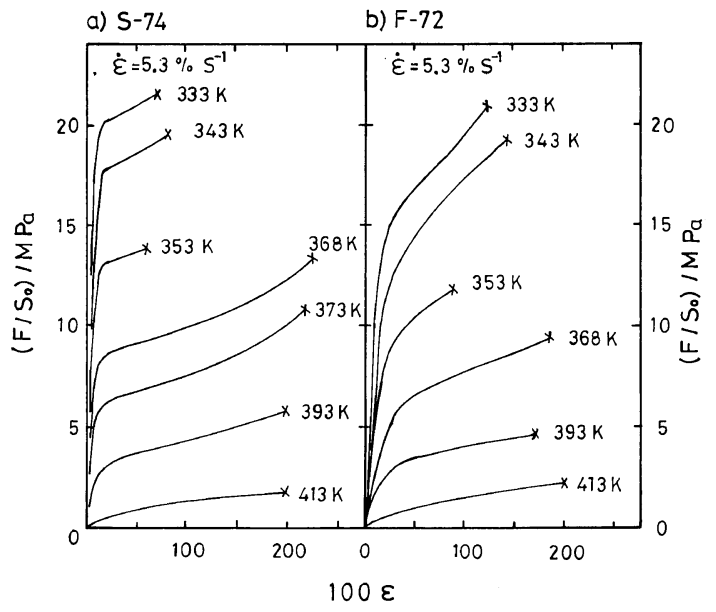

Figure 7. Stress-strain curves for (a) semi-IPN (S-74). and (b) full-IPN (F-72) obtained at various temperatures indicated. Strain rate $\dot{\varepsilon}$ was $5.3 \% \mathrm{~s}^{-1}$. Marks $(\times)$ denote break points.

Figures $7 \mathrm{a}$ and $7 \mathrm{~b}$ show the stress-strain curves for S-74 and F-72 obtained at a strain rate $\dot{\varepsilon}$ of $5.3 \% \mathrm{~s}^{-1}$ at various temperatures. It can be seen that, at temperatures below the $T_{\mathrm{g}}$ of PMMA, the curves for both specimens exhibit behavior typical of hard and strong plastics. ${ }^{21}$ The initial slope of the 


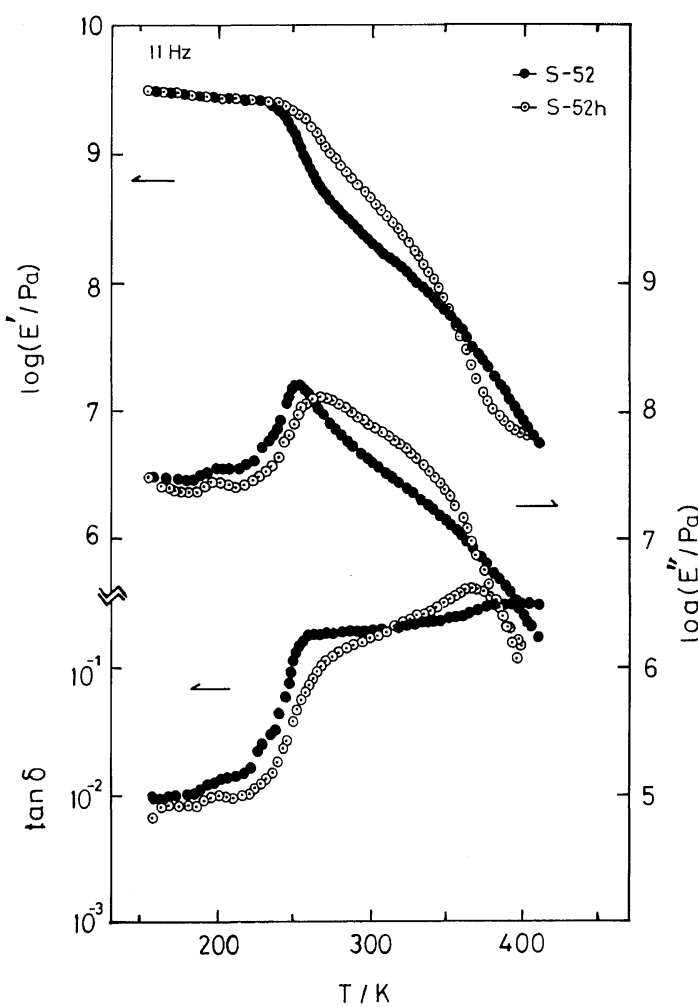

Figure 8. Storage $\left(E^{\prime}\right)$, loss $\left(E^{\prime \prime}\right)$ moduli, and loss tangents $(\tan \delta)$ at $11 \mathrm{~Hz}$ versus temperature for semiIPNs, S-52 and corresponding S-52h with higher crosslinking density of the NBR network, respectively. Measurements were carried out at a heating rate of $1 \mathrm{~K}$ $\min ^{-1}$.

curve for S-74 is larger than that for F-72. On the other hand, at temperatures above the $T_{\mathrm{g}}$ of PMMA, the behavior of soft material is seen for both specimens.

Effect of Crosslinking Density of NBR Network. It was reported for SIPNs that the domain size of the second component becomes smaller with an increase in crosslinking density $v$ of the first network. ${ }^{1,3,4}$ Thus, we studied the effect of $v$ by reexamining the dynamic viscoelastic behavior of two semi-IPNs in which the first network had different crosslinking densities. One sample was S52 with $v=1.4 \times 10^{2} \mathrm{~mol} \mathrm{~m}^{-3}$ and the other sample consisted of an NBR network with $v=5.1 \times 10^{2}$ mol $\mathrm{m}^{-3}$ but had nearly the same composition as S52. This specimen was coded as $\mathrm{S}-52 \mathrm{~h}$.

Figure 8 shows the $E^{\prime}, E^{\prime \prime}$, and $\tan \delta$ curves for S52 and S-52h. The $E^{\prime}$ curve for S-52h shows a gradual decrease over the temperature range from 250 to $400 \mathrm{~K}$, and that for S-52 exhibits a two-step decrease. The $\tan \delta$ curve for $\mathrm{S}-52 \mathrm{~h}$ exhibits a distinct peak at $370 \mathrm{~K}$ with a shoulder on the lower temperature side, but that for S-52 is constant over the temperature range from 250 to $400 \mathrm{~K}$. These differences imply that the extent of molecular mixing of NBR and PMMA components is greater in S$52 \mathrm{~h}$ than that in S-52.

\section{DISCUSSION}

Sperling et al. ${ }^{7}$ reported that for SIPNs consisting of incompatible polymer pairs such as poly(styreneco-butadiene) and polystyrene, the extent of molecular mixing of the two components is larger in fullIPNs than in semi-IPNs. Obviously, this conclusion applies to the present NBR/PMMA systems. We arrived at a similar conclusion for SIPNs consisting of a semi-compatible pair poly(ethyl acrylate)/ PMMA. ${ }^{5}$ From the results on these two systems, it follows that the most essential factor controlling the morphology of SIPNs is the structure of the first network. Whether the two constituent polymers are compatible or not appears unimportant for determining the morphology, except in such a system as a complex-forming IPN $^{6}$ in which strong and specific interactions act between the two components.

In the previous paper, ${ }^{5}$ we proposed that in the early stage of the reaction, microgels of the second component are formed in large pools of the reaction mixture containing the second monomer, crosslinking agent, and initiator, and are eventually interconnected to form an IPN having microheterogeneous but essentially mutually interpenetrating continuous phases. In full-IPNs, the crosslinks in each phase prevent subsequent phase separation. On the other hand, in semi-IPNs the uncrosslinked polymer component may eventually be phaseseparated from the crosslinked component. However, the size of the microdomains obviously depends on the crosslink density of the first network and to some extent on the incompatibility of the two components. The higher the crosslink density and the more strongly compatible the two components, the smaller the microdomains of the second component become.

Acknowledgment. One of the authors (T.K.) 
wishes to express his appreciation for the support provided by the Ministry of Education, Science and Culture (Mombusho) under a Grant-in-Aid for Scientific Research No. 543026.

\section{REFERENCES}

1. A. A. Donateli, D. A. Thomas, and L. H. Sperling, "Recent Advances in Polymer Blends, Grafts and Blocks," L. H. Sperling, Ed., Plenum Press, New York, 1974.

2. H. L. Frisch, K. C. Frisch, and D. Klempner, "Chemistry and Properties of Crosslinked Polymers," S. S. Labana, Ed., Academic Press, New York, 1976, p 205.

3. J. A. Mason and L. H. Sperling, "Polymer Blends and Composites," Plenum Press, New York, 1976.

4. L. H. Sperling, J. Polym. Sci., Macromol. Rev., 12, 141 (1977)

5. H. Adachi and T. Kotaka, Polym. J., 14, 379 (1982).

6. H. Adachi, S. Nishi, and T. Kotaka, Polym. J., 15, 985 (1983).

7. A. A. Donatelli, L. H. Sperling, and D. A. Thomas, Macromolecules, 9, 671 (1976); ibid., 9, 676 (1976).

8. H. Adachi, K. Adachi, and T. Kotaka, Polym. J., 12, 329 (1980).

9. M. Morton, "Science and Technology of Rubber,"
F. R. Eirich, Ed., Academic Press, New York, 1978, p 66.

10. K. Kato, J. Polym. Sci., B4, 35 (1966).

11. C. K. Riew and R. W. Smith, J. Polym. Sci., A, 9, 2739 (1971).

12. D. Kaplan, J. Appl. Polym. Sci., 20, 2615 (1976).

13. G. Kraus and K. W. Rollman, "Multicomponent Polymer Systems," Vol. 99, N. J. Platzer, Ed., Adv. Chem. Ser., American Chemical Society, Washington, D. C., 1971, p 189.

14. W. E. A. Davies, J. Phys. D, Appl. Phys., 4, 1176 (1971).

15. L. E. Nielsen, "Predicting the Properties of Mixtures," Marcel Dekker, Inc., New York, 1978.

16. D. R. Paul, "Polymer Blends," Vol. 1, D. R. Paul and S. Newman, Ed., Academic Press, New York, 1978, p 12.

17. M. Takayanagi, J. Appl. Polym. Sci., 10, 113 (1966).

18. G. Allen, M. J. Bowden, S. M. Todd, D. J. Blundell, G. M. Jeffs, and W. E. A. Davies, Polymer, 15, 28 (1974).

19. L. E. Nielsen, "Mechanical Properties of Polymers and Composites," Vol. 2, Marcel Dekker, New York, 1974, p 365.

20. J. D. Fery, "Viscoelastic Properties of Polymers," 3rd ed, John Wiley, New York, 1980.

21. T. S. Carswell and H. K. Nason, Mod. Plast., 21, 121 (1944). 